ISSN 2145-6054

\title{
EL DEBIDO PROCESO ADMINISTRATIVO EN EL TRÁMITE DE LA CALIFICACIÓN DE LA PÉRDIDA DE CAPACIDAD LABORAL $^{1}$
}

\author{
Administrative Due Process in the Procedure of Rating the \\ Loss of Working Capacity ${ }^{1}$
}

\author{
Iliana Marcela Fortich Lozano ${ }^{2}$ \\ Yezid Carrillo De la Rosa ${ }^{3}$
}

Fecha de Recepción: Mayo 5 de 2014

Fecha de Aceptación: Mayo 12 de 2014

SUMARIO: 1. Introducción; 2. El derecho fundamental al debido proceso; 3. El principio del debido proceso administrativo; 4 . El respeto del derecho fundamental al debido proceso en el trámite de la calificación de la pérdida de capacidad laboral; 4.1. Juntas de calificación de invalidez; 4.2. Procedimiento para la calificación de la pérdida de la capacidad laboral; 4.3. Respeto al debido proceso en la calificación de la pérdida de la capacidad laboral; 5. Análisis estático del precedente judicial del debido proceso en el proceso de calificación de la pérdida de la capacidad laboral; 5.1. Análisis estático de precedente judicial: Violación del debido proceso cuando se niega recurso de apelación por falta de requisitos formales; 5.2. Análisis estático de precedente judicial: vulneración del debido proceso por indebida notificación de los actos de carácter particular; 5.3. Análisis estático de precedente judicial: vulneración del debido proceso por desconocimiento de la historia clínica; 6. Conclusiones; 7. Referencias bibliográficas.

\footnotetext{
${ }^{1}$ Avance del proyecto: la seguridad social como protección de los trabajadores en Colombia, línea de investigación: derechos fundamentales y derechos sociales. Proyecto iniciado en el año 2014. Investigador principal: Iliana Fortich Lozano, director del proyecto: Yezid Carrillo De la rosa. Proyecto avalado por la Universidad de Cartagena y la Universidad libre seccional Cartagena.

${ }^{2}$ Docente Universidad Libre Sede Cartagena, Docente Corporación Universitaria Rafael Núñez CURN, Coinvestigadora del grupo de Investigación: Teoría Jurídica y Derechos Fundamentales Phronesis, Abogada, Corporación Universitaria de la Costa CUC, Conciliadora de la Universidad Libre, Especialista en Seguridad Social, Universidad de Cartagena, Candidata Magíster en Derecho Universidad de Cartagena.Email: ilianafortich@hotmail.com.

${ }^{3}$ Docente Universidad de Cartagena, Docente Universidad Libre Sede Cartagena, Docente Corporación Universitaria Rafael Núñez CURN, Director del grupo de Investigación, Teoría Jurídica y Derechos Fundamentales Phronesis, Abogado Universidad Nacional, licenciado en Filosofía y letras Universidad Santo Tomas, Especialista en Derecho Público Universidad Externado de Colombia, Especialista en Ética y Filosofía Política Universidad de Cartagena, Magíster en Derecho Universidad Nacional, Candidato a Doctor en Derecho Universidad Externado de Colombia.Email:yezidcarrillo@hotmail.com.
} 


\section{COMO SE CITA ESTE ARTÍCULO (APA 6)}

Fortich Lozano, I. M., Carrillo De la Rosa, Y. (2014) El debido Proceso Administrativo en el trámite de la calificación de la pérdida de capacidad laboral. (Y. Carrillo De la rosa, Ed.) Revista Jurídica Mario Alario D'Filippo, VI (12), pág. 57-69

\section{RESUMEN}

Este avance de investigación tiene como propósito abordar las implicaciones y efectos que el derecho fundamental al debido proceso tiene en el procedimiento de la calificación de la pérdida de la capacidad laboral. Para ello, se analiza el significado que el debido proceso y la aplicación que dicho principio tiene en el ámbito de las actuaciones de la administración pública, así como las garantías normativas y jurisprudenciales que conlleva el principio del debido proceso en el procedimiento de la calificación de la pérdida de la capacidad laboral.

\section{ABSTRACT}

This advance research aims to address the implications and effects that the fundamental right to due process is in the process of rating the loss of working capacity. For this, the meaning is analyzed that due process and applying that principle has in the scope of the actions of public administration as well as regulatory and jurisprudential guarantees that carries the principle of due process in the procedure qualification the loss of working capacity.

\section{PALABRAS CLAVE}

Debido proceso administrativo, junta de calificación, pensión de invalidez, seguridad social, jurisprudencias constitucionales.

\section{KEYWORDS}

Due administrative process, rating board, invalidity pension, social security, constitutional jurisprudence. 


\section{INTRODUCCIÓN}

Este avance de investigación tiene como propósito abordar las implicaciones y efectos que el derecho fundamental al debido proceso tiene en el procedimiento de la calificación de la pérdida de la capacidad laboral. Para ello inicialmente se analizará el significado que el debido proceso tiene en nuestro ordenamiento jurídico constitucionalizado. En segundo lugar la aplicación que dicho principio tiene en el ámbito de las actuaciones de la administración pública. En tercer lugar las garantías que conlleva el principio del debido proceso en el procedimiento de la calificación de la pérdida de la capacidad laboral. Finalmente se realizará el análisis estático de precedente judicial de tres jurisprudencia que abordan la cuestión de la violación del debido proceso en el procedimiento específico que nos ocupa en este escrito.

\section{MATERIALES Y MÉTODOS}

Se trata de un avance de investigación jurídica pura (doctrina y jurisprudencia) que combina el análisis hermenéutico de textos jurisprudenciales y legales.

El proceso metodológico que se implementó en la búsqueda de información fue el de recuperación bibliográfica, y el estudio de sentencias expedidas por la Corte Constitucional, la descripción y análisis del material con temas relacionados a la problemática en estudio. La consulta a diversas fuentes permitió organizar una guía de contenido en la que se han tratado los aspectos teóricos generales que desde el punto de vista legal, jurisprudencial y conceptual hacen referencia a la temática.

\section{RESULTADOSYDISCUSIÓN}

En este avance de investigación lo que plantea la discusión, es saber si realmente las Juntas de calificación de invalidez, cumplen con el debido proceso administrativo, al momento de evaluar a los afiliados al Sistema de Seguridad Social Integral, cuando les sucede un accidente común, o relacionado con la labor que realizan, o se les degenera una enfermedad de origen común, o de tipo laboral.

Lo que nos interesa en este estudio es saber si realmente esta valoración de la pérdida de la capacidad laboral, es realizada bajo los parámetros que nos señala el Artículo 29 de la Constitución Nacional, de cómo deben realizarse las actuaciones administrativas, sin violar ninguno de los pasos del debido proceso, que permita una calificación justa y legal para los afiliados que les toca acudir a este procedimiento para obtener una pensión de invalidez si llegasen a tener una pérdida del $50 \%$ de su capacidad laboral.

\section{EL DERECHO FUNDAMENTAL AL DEBIDO PROCESO}

El derecho fundamental al debido proceso se encuentra consagrado en el Artículo 29 de la Constitución Política y prescribe que este debe ser tenido en cuenta y aplicado a todas las actuaciones judiciales y administrativas; por consiguiente, cualquier tipo de actuaciones judiciales 
y administrativas deben desarrollarse con respeto de las garantías inherentes al derecho fundamental del debido proceso. La Corte ha definido el debido proceso "como el conjunto de garantías previstas en el ordenamiento jurídico, a través de las cuales se busca la protección del individuo incurso en una actuación judicial o administrativa, para que durante su trámite se respeten sus derechos y se logre la aplicación correcta de la justicia." (Corte Constitucional de Colombia, 2010)

El derecho fundamental al debido proceso se encuentra protegido en el Derecho Internacional por una serie de instrumentos tales como la Declaración Universal de Derechos Humanos (Art. 10 y 11), la Declaración Americana de Derechos y Deberes del Hombre (Art. XVIII y XXVI), El Pacto Internacional de Derechos Civiles y Políticos (Art.14 y 15), y la Convención Americana sobre Derechos Humanos (Art.8). También cuenta con un amplio desarrollo jurisprudencial llevado a cabo por órganos internacionales, en la que sobresale la Corte Interamericana de Derechos Humanos (Corte Constitucional de Colombia, 1996), (Corte Constitucional de Colombia, 1997), (Corte Constitucional de Colombia, 1999), (Corte Constitucional de Colombia, 2000), (Corte Constitucional de Colombia, 2001), (Corte Constitucional de Colombia, 2002), (Corte Constitucional de Colombia, 2003), (Corte Constitucional de Colombia, 2003), (Corte Constitucional de Colombia, 2005).

La jurisprudencia (corte constitucional de colombia, 1993), (corte constitucional de colombia, 1996), (corte constitucional de colombia, 2005) por vía de interpretación ha establecido que el debido proceso incluye al menos las siguientes garantías:

a. La garantía de acceso libre y en igualdad de condiciones a la justicia, con el fin de lograr una pronta resolución judicial y el derecho a la jurisdicción.

b. La garantía de juez natural.

c. Las garantías inherentes a la legítima defensa.

d. La determinación y aplicación de trámites y plazos razonables.

e. La garantía de imparcialidad.

A continuación puntualizaremos cuales son las características o aspectos relevantes del debido proceso aplicado a las actuaciones administrativas y en especial el efecto que dicho derecho tiene en el trámite de la calificación de la pérdida de la capacidad laboral.

\section{ELPRINCIPIO DEL DEBIDO PROCESO ADMINISTRATIVO}

En relación con el debido proceso en las actuaciones administrativas la jurisprudencia constitucional (Corte Constitucional de Colombia, 1998), (Corte Constitucional de Colombia, 2001), (Corte Constitucional de Colombia, 2002), (Corte Constitucional de Colombia, 2003), (Corte Constitucional de Colombia, 2004), (Corte Constitucional de Colombia, 2005), (Corte Constitucional de Colombia, 2005), (Corte Constitucional de Colombia, 2005), (Corte 
Constitucional de Colombia, 2005),(Corte Constitucional de Colombia, 2005) ha establecido que los principios que conforman este derecho fundamental deben ser respetados por las actuaciones que desarrolle la administración pública en el cumplimiento de sus funciones y realización de sus objetivos y fines, de manera que se garantice:

a. El acceso a procesos justos y adecuados.

b. El principio de legalidad y las formas administrativas previamente establecidas.

c. Los principios de contradicción e imparcialidad.

d. Los derechos fundamentales de los asociados.

La garantías constitucionales incluidas en el debido proceso permiten un correcto, razonable y justo ejercicio de la función pública administrativa acorde con la Constitución, las leyes y los reglamentos vigentes y evita actuaciones abusivas o arbitrarias, por parte de la administración pública, mediante la expedición de actos administrativos que resulten lesivos de derechos o contrarios a los principios del Estado de Derecho (T-442-92, T-120-93, T-020-98, T-386-98, T-1013-99, T-009-00, T-1739-00, T165-01, T-772-03, T-746-05 y C-1189-05).

Los argumentos anteriores permiten hablar de un principio del debido proceso administrativo que trae importantes consecuencias para los asociados como para la administración. En relación con los asociados:

a. Conocer las actuaciones de la administración.

b. Pedir y controvertir las pruebas.

c. Ejercer con plenitud su derecho de defensa.

d. Impugnar los actos administrativos.

e. Gozar de las demás garantías establecidas en su beneficio.

En lo que respecta a la administración, la más importante consecuencia es que todas las manifestaciones del ejercicio de la función pública administrativa se encuentran cobijadas por el debido proceso, tales como:

a. La formación y ejecución de actos administrativos.

b. Las peticiones presentadas por los particulares.

c. Los procesos que se adelanten contra la administración por los ciudadanos en ejercicio legítimo de su derecho de defensa. 


\section{EL RESPETO DEL DERECHO FUNDAMENTAL AL DEBIDO PROCESO EN EL TRÁMITE DE LA CALIFICACIÓN DE LA PÉRDIDA DE CAPACIDAD LABORAL}

\subsection{JUNTAS DE CALIFICACIÓN DE INVALIDEZ}

Según el Artículo 142 del Decreto-Ley 019 de 2012 los responsables y legalmente facultados para efectuar la calificación de invalidez son la Administradora Colombiana de Pensiones Colpensiones, Administradoras de Riesgos Laborales ARL, Empresas Promotoras de Salud EPS y las Aseguradoras y, por otra parte, que es el tema que nos ocupa, las juntas de calificación de invalidez, creadas por la Ley 100 de 1993.

Las Juntas Regionales y Nacional de Calificación de Invalidez, son organismos del Sistema de Seguridad Social del orden nacional, de creación legal, adscritas al Ministerio del Trabajo, con Personería Jurídica de Derecho privado, sin ánimo de lucro, de carácter interdisciplinario, sujetas a revisoría fiscal, con autonomía técnica y científica en los dictámenes periciales. Sus integrantes son designados por el gobierno, pero no tienen el carácter de servidores públicos, sus miembros tienen derecho al pago de unos honorarios que son cancelados por las entidades del sistema que requieran sus servicios. Los dictámenes de las juntas no son actos administrativos, pero pueden ser controvertidos por la justicia ordinaria laboral.

\subsection{PROCEDIMIENTO PARA LA CALIFICACIÓN DE LA PÉRDIDA DE LA CAPACIDAD LABORAL}

El Artículo 48 de la C.P. consagró la seguridad social como un derecho irrenunciable que debe garantizarse a todos los habitantes del territorio colombiano y lo organizó como un servicio público obligatorio bajo "la dirección, coordinación y control" del Estado que debe ser prestado con sujeción a los principios de solidaridad, eficacia y universalidad.

En congruencia con el Estatuto Superior, la Ley 100 de 1993 diseñó un nuevo modelo de seguridad social en Colombia no solo se unifican los regímenes normativos existentes, sino que también se propicia una dinámica de gestión que combina lo público y lo privado, en un Sistema Integral de Seguridad Social que ampara de forma anticipada a los ciudadanos, contra determinadas contingencias que puedan presentarse en el desarrollo de la vida laboral y, en el desenvolvimiento de la vida misma.

El Sistema General de Riesgos Laborales -SGRL-, dispone la protección del trabajador respecto de los riesgos derivados del trabajo. La legislación del Sistema de Riesgos Laborales prevista entre otras disposiciones en la Ley 100 de 1993, el Decreto 1295 de 1994, la Ley 776 de 2002 y la Ley 1562 de 2012, lo define como "un conjunto de entidades públicas y privadas, normas y procedimientos, que tiene la finalidad de prevenir, proteger y atender las consecuencias que se derivan de los riesgos profesionales, es decir, de los accidentes y las enfermedades que puedan padecer las personas por causa o con ocasión del trabajo". (Decreto 1295 de 1994)

En virtud de la finalidad perseguida por el Sistema de Riesgos Laborales, las normas que lo regulan 
consagran la noción legal de accidente de trabajo y enfermedad profesional, con elementos conceptuales que permiten identificar si la situación de hecho que se analiza corresponde o no a un evento relacionado con la actividad laboral o profesional del afiliado.

Al respecto, la normativa de riesgos laborales dispone que cuando ocurre un accidente laboral o enfermedad laboral, el afiliado tendrá derecho a recibir:

a. El servicio asistencial de salud correspondiente, con cargo al sistema, e igualmente.

b. Las prestaciones económicas, que se determinarán de acuerdo a las secuelas de la enfermedad o el accidente, cómo incapacidades temporales, subsidios por incapacidad temporal, incapacidad permanente parcial o pensión de invalidez según la gravedad de la pérdida de capacidad laboral; en caso de muerte los beneficiarios del afiliado tendrán derecho a pensión de sobrevivientes y al denominado auxilio funerario. (Corte Constitucional de Colombia, 2011),(Corte Constitucional de Colombia, 2008)

c. De igual forma, si a un afiliado al Sistema General de Seguridad Social integral, llegare a ocurrirle un accidente o una enfermedad que no guardara relación con el trabajo el afiliado también tendrá derecho a que el sistema le realice la evaluación pertinente para determinar la pérdida de la capacidad laboral.

Para establecer si una persona tiene derecho al reconocimiento de alguna de las prestaciones asistenciales o económicas, se requiere de la calificación de la pérdida de capacidad laboral, entendida como un mecanismo que permite fijar el porcentaje de afectación del "conjunto de las habilidades, destrezas, aptitudes y/o potencialidades de orden físico, mental y social, que le permiten al individuo desempeñarse en un trabajo habitual" (Decreto 917 de 1999, Artículo 2). El derecho a la valoración de la disminución de dicha capacidad se encuentra regulado básicamente en las mismas leyes y decretos que desarrollan el SGRL, con mayor énfasis en la Ley 100 de 1993, el Decreto 917 de 1999 y el Decreto 2463 de 2001 en donde se establece el procedimiento respectivo.

El Artículo 250 de la Ley 100 de 1993, señala que la calificación de pérdida de capacidad laboral por accidente de trabajo o enfermedad laboral debe ajustarse a las mismas reglas y procedimientos establecidos para la valoración de pérdida de capacidad laboral para el caso de padecimientos por riesgo común, lo que significa que la calificación respecto de la pérdida de capacidad laboral se lleva a cabo independientemente de que la causa sea laboral o común.

La jurisprudencia de la Corte Constitucional Colombiana ha establecido que la calificación de la pérdida de capacidad laboral es un derecho que tiene toda persona, y que constituye el fundamento o medio para acceder a la garantía y protección de otros derechos fundamentales como la salud, la seguridad social y el mínimo vital, pues es a través de él que se establece qué tipo de prestaciones tiene derecho el afiliado que resulte afectado por una enfermedad o accidente, producido con ocasión o como consecuencia de la actividad laboral, o por causas de origen común. El procedimiento es el siguiente: 
a. Las entidades competentes para realizar, en primera oportunidad, la valoración de la pérdida de la capacidad laboral son Administradora Colombiana de Pensiones Colpensiones, Administradoras de Riesgos Laborales ARL, Empresas Promotoras de Salud EPS y las Aseguradoras. Todos estos entes deben expedir los actos de calificación expresando los fundamentos de hecho y de derecho que los llevó a tomar la decisión final y, además, deben informar los recursos que proceden en contra de la decisión tomada.

b. Si el afiliado no está conforme con la calificación emitida por alguna de estas entidades, podrá presentar un escrito de inconformidad, dentro de los diez (10) días siguiente, donde solicita se le remita a la junta regional de invalidez para una nueva valoración quienes calificarán en primera instancia.

c. Si surge alguna inconformidad con este nuevo dictamen podrá el afiliado o la entidad que emitió el primer dictamen de calificación apelar en segunda instancia dentro de los cinco (5) días siguientes a la calificación, y el afilado deberá ser sometido a una nueva valoración por la junta nacional de calificación de invalidez en segunda instancia, quien decidirá dentro de los cinco (5) días siguientes. Contra estas decisiones proceden las acciones legales.

d. Los recursos mediante los cuales el peticionario puede manifestar su inconformidad con las decisiones tomadas se encuentran en los Artículo 33 y 34 del Decreto 2463 de 2001 y corresponden al recurso de reposición y al recurso de apelación.

Es importante señalar que la inobservancia de los preceptos legales que regulan valoración de pérdida de capacidad laboral, la negativa por parte de las entidades obligadas a ello a realizar la valoración de la persona cuando su situación de salud lo requiere, constituyen una flagrante vulneración del derecho a la seguridad social consagrado en el Artículo 48 superior, e igualmente se erigen en barreras de acceso a las garantías fundamentales a la salud, la vida digna y al mínimo vital, al no permitir determinar el origen de la afección, el nivel de alteración de la salud y de la pérdida de capacidad laboral del trabajador.

\subsection{RESPETO AL DEBIDO PROCESO EN LA CALIFICACIÓN DE LA PÉRDIDA DE LA CAPACIDAD LABORAL}

El Artículo 3 del Decreto 1352 de 2013 establece que el procedimiento y la actuación de los miembros de las Juntas de calificación de Invalidez estarán regidos por una serie de principios de rango constitucional entre los cuales incluye el debido proceso.

En la sentencia T-108-07, la corte expresa que:

"Durante este trámite, tal como lo ha señalado la Corte, el interesado tiene los derechos propios de todo interviniente en una actuación administrativa, y, especialmente, el derecho a que se dé la oportunidad de controvertir la calificación o valoración médica relativa a la disminución de su capacidad laboral, tal y como se encuentra previsto en los Artículos 11, 35 y 40 del Decreto 2463 de 2001. Lo anterior, constituye la materialización del derecho al debido proceso, el cual, de acuerdo con la jurisprudencia constitucional, debe ser respetado durante el trámite que se sigue por estas entidades." 
De igual forma, en la sentencia de la (corte constitucional de colombia, 2011) se afirmó que:

\begin{abstract}
"El cumplimiento de las normas que regulan la adopción de decisiones por parte de las juntas de calificación de invalidez o las juntas o tribunales médicos de la Policía Nacional o de las Fuerzas Militares son parte integrante del derecho fundamental al debido proceso de las personas que están surtiendo los trámites para la determinación de su porcentaje de pérdida de la capacidad laboral".
\end{abstract}

De lo anteriormente expuesto, se puede inferir como sub-regla o precedente constitucional que: quien se encuentre dentro de un proceso de calificación de pérdida de capacidad laboral tiene derecho a acceder a todos los mecanismos que han sido otorgados por la legislación para proteger su derecho al debido proceso.

\title{
4. ANÁLISIS ESTÁTICO DEL PRECEDENTE JUDICIAL DEL DEBIDO PROCESO EN EL PROCESO DE CALIFICACIÓN DE LA PÉRDIDA DE LA CAPACIDAD LABORAL
}

\subsection{ANÁLISIS ESTÁTICO DE PRECEDENTE JUDICIAL: VIOLACIÓN DEL DEBIDO PROCESO CUANDO SE NIEGA RECURSO DE APELACIÓN POR FALTA DE REQUISITOS FORMALES}

En la Sentencia de la (corte constitucional de colombia, 2013) se aborda el caso de Rosa Elvira Bermúdez, mediante apoderada judicial, interpuso acción de tutela para solicitar el amparo de sus derechos fundamentales a la seguridad social y el debido proceso que considera vulnerados por la Junta Regional de Calificación de Invalidez de Antioquia. La accionante, de 73 años, es afiliada al SISBEN y quien se encuentra en una situación de debilidad manifiesta (Padece de lumbago no especificado, hipertensión esencial (primaria), hipotiroidismo no especificado, espondilopatía no especificada y un tumor benigno de encéfalo). La actora acude al Instituto de Seguros Sociales para evaluar su grado de invalidez quien emitió un dictamen de calificación de invalidez en el cual determinó que la actora ostentaba una pérdida de capacidad laboral del 35.54\%. Posteriormente, la peticionaria interpuso recurso de apelación contra la resolución emitida por el Instituto de Seguros Sociales. Dicho recurso fue resuelto por la Junta Regional de Calificación de Invalidez de Antioquia quienes, el 30 de agosto de 2011, establecieron una pérdida de capacidad laboral del $45.14 \%$. El 9 de septiembre de 2011, la ciudadana presentó una carta informal ante la Junta de Calificación de Invalidez de Antioquia en la cual manifestaba su inconformidad con el dictamen emitido y solicitó su revisión. En respuesta a lo anterior, la Junta Regional de Calificación de Invalidez expidió una resolución en la cual confirmó el porcentaje atacado y especificó que no procedía el recurso de apelación debido a que no fue solicitado, de manera expresa, por la peticionaria.

En virtud de los anteriores hechos la Corte considera como problema jurídico la necesidad de determinar si la Junta Regional de Calificación de Invalidez de Medellín vulneró los derechos fundamentales al debido proceso y a la seguridad social en pensiones de la señora Rosa Elvira Bermúdez al haber omitido el envío de su dictamen de calificación de pérdida de capacidad laboral ante la Junta Nacional de Calificación de Invalidez, por considerar que, dentro del escrito presentado por la actora, no se manifestó de forma expresa la interposición del recurso de apelación. 
Para resolver este problema la Corte señala que tratándose de los recursos de reposición y apelación en contra de las decisiones tomadas por las juntas de calificación de invalidez, los ciudadanos tienen la posibilidad de presentarlos en los tiempos determinados por la ley y sin ningún tipo de formalidades específicas pues, únicamente se exige que estos expresen y argumenten las razones de su desacuerdo. Esto es conforme a lo que establece la norma que regula tal procedimiento que señala que "el recurso de apelación podrá interponerse directamente sin que se requieran formalidades especiales, señalando los motivos de inconformidad y acreditando las pruebas que se pretendan hacer valer".

Según la Corte el escrito presentado por la solicitante pudo haber sido interpretado de dos formas.

"La primera se refiere a que, dentro del escrito no se solicitó de manera expresa el recurso de apelación y, únicamente, podía tomarse como la interposición de un recurso de reposición que debía ser resuelto por la misma entidad-interpretación adoptada por la parte accionada-. La segunda que, conforme a la informalidad que se predica del proceso de interposición del recurso de apelación contra las decisiones tomadas por las juntas de calificación de invalidez, el escrito presentado debió entenderse como un recurso de apelación y, de esta forma, continuaba con su revisión por parte de la Junta Nacional de Calificación de Invalidez. Considera la Sala de Revisión que, en aras del amparo al debido proceso que ostenta la actora, la Junta Regional de Calificación de Invalidez de Antioquia debió optar por la segunda interpretación pues resulta acorde con los lineamientos establecidos por la jurisdicción Constitucional y permite una protección más amplia al derecho fundamental aludido".

De esta forma considera la Corte desproporcionado y violatorio del derecho fundamental al debido proceso el impedir que la solicitante cuente con la oportunidad de que su dictamen de pérdida de capacidad laboral sea revisado por la Junta Nacional de Calificación de Invalidez.

\subsection{ANÁLISIS ESTÁTICO DE PRECEDENTE JUDICIAL: VULNERACIÓN DEL DEBIDO PROCESO POR INDEBIDA NOTIFICACIÓN DE LOS ACTOS DE CARÁCTER PARTICULAR}

En la Sentencia de la (corte constitucional de colombia, 2011) aborda el problema de saber si se una entidad administradora de fondos de pensiones (Instituto de Seguros Sociales - Seccional Atlántico) vulnera los derechos fundamentales al mínimo vital, al debido proceso y a la seguridad social, de uno de sus afiliados (Pablo Antonio Caicedo Mora), al negarse a revisar el dictamen de calificación de su pérdida de capacidad laboral, en el que se estableció una fecha de estructuración de invalidez en forma retroactiva, fundamentando su decisión en que el interesado no interpuso los recursos procedentes en la oportunidad legal y que la decisión quedó en firme, sin tener en cuenta que este manifiesta que en el escrito mediante el cual se le comunicó el resultado del dictamen no se le informó la fecha de estructuración de su invalidez y que para esa época conservaba su capacidad laboral.

Para resolver el problema la Corte señala que de conformidad con el Artículo 29 de la Constitución Política las actuaciones que adelante el Estado para resolver una solicitud de reconocimiento de un derecho o prestación, deben adelantarse respetando, entre otras, las garantías del peticionario al derecho de defensa y de impugnación y publicidad de los actos administrativos. Una de las formas 
de respetar dichas garantías, es a través de la notificación de las actuaciones administrativas que está regulada en los Artículos 44 al 48 del Código Contencioso Administrativo que establece el procedimiento y los requisitos sin los cuales se entenderá que esta no se surtió y la decisión no producirá efectos legales. Ello porque en los eventos en los que una entidad pública notifica indebidamente una decisión, le impide al interesado ejercer su derecho de defensa y vulnera su derecho fundamental al debido proceso. Por las anteriores razones señala la corte que:

\begin{abstract}
"todo dictamen de calificación de la pérdida de capacidad laboral debe ser notificado personalmente al afiliado calificado, porque las decisiones que se toman en ese tipo de actos son esenciales para determinar si el afiliado tiene o no derecho a la pensión de invalidez, y, por lo tanto, se le debe garantizar su derecho al debido proceso, brindándole la oportunidad de controvertir la decisión ante la Junta Regional de Calificación de Invalidez, así como recurrir la decisión que esta entidad adopte ante la Junta Nacional de Calificación de Invalidez en caso de que sea contraria a sus intereses."
\end{abstract}

\title{
4.3. ANÁLISIS ESTÁTICO DE PRECEDENTE JUDICIAL: VULNERACIÓN DEL DEBIDO PROCESO POR DESCONOCIMIENTO DE LA HISTORIA CLÍNICA
}

En la Sentencia (corte constitucional de colombia, 2009) la corte aborda el problema de si la Junta Regional de Calificación de Invalidez de Bogotá y Cundinamarca violó el derecho fundamental al debido proceso de Horacio Dueñas al indicar como fecha de estructuración de su invalidez el veintiséis (26) de julio de 2004 con supuesto desconocimiento de su historia clínica y ausencia de motivación. Ello porque las pretendidas vulneraciones a los derechos fundamentales a la vida, a la dignidad humana y a la seguridad social tienen su origen en lo anterior, pues la razón por la cual el ISS se negó a reconocerle al señor Dueñas la pensión de invalidez se basó, precisamente, en el dictamen de la Junta demandada que se acusa de violatorio del debido proceso.

Para dar solución a este problema la Corte señala, en primer lugar, que los dictámenes emitidos por las juntas de calificación de invalidez debe ser motivados, en el sentido de manifestar las razones que justifican en forma técnico-científica la decisión (Artículo 31 del Decreto 2463 de 2001). En segundo lugar, que la jurisprudencia constitucional ha señalado entre otras reglas que:

\begin{abstract}
"Los dictámenes que emitan las juntas de calificación, deben contener expresamente los fundamentos de hecho y de derecho que dieron origen a esta decisión [según el Artículo $9^{\circ}$ del Decreto 2463 de 2001 que] (...) indica que los fundamentos de hechos son todos aquellos que se relacionan con la ocurrencia de determinada contingencia, lo cual incluye historias clínicas, reportes, valoraciones o exámenes médicos periódicos; y en general, los que puedan servir de prueba para certificar una determinada relación causal, tales como certificado de cargos y labores, comisiones, realización de actividades, subordinación, uso de determinadas herramientas, aparatos, equipos o elementos, contratos de trabajo, estadísticas o testimonios, entre otros, que se relacionen con la patología, lesión o condición en estudio y que los fundamentos de derecho son todas las normas que se aplican al caso de que se trate".
\end{abstract}

En tercer lugar reseña una serie de decisiones anteriores en los que se han estudiado situaciones que configuran violaciones del derecho al debido proceso por parte de las juntas de calificación de 
invalidez como en la Sentencia T-859 de 2004 en la que se cuestionó la fecha de la estructuración de la invalidez establecida por una junta de calificación por haber sido determinada sin tener en cuenta las pruebas; la sentencia (corte constitucional de colombia, 2005), en la que se estimó que una junta de calificación había vulnerado el derecho al debido proceso al fijar el porcentaje de pérdida de capacidad laboral pues pretermitió algunas partes del procedimiento reglamentario y existían falencias en la motivación; la sentencia (corte constitucional de colombia, 2007), ocasión en la que la junta demandada tampoco tuvo en cuenta todas las patologías que sufría el peticionario ni ofreció sustentación alguna respecto de su exclusión; y la sentencia (Corte Constitucional de Colombia, 2008) en la que se consideró que el hecho de no tener en cuenta todos los exámenes médicos realizados al actor para determinar el porcentaje de la incapacidad laboral y no justificarlo desconoce el derecho al debido proceso. Además, recordó a la junta accionada que, en caso de no tener certeza sobre el diagnóstico de la accionante, la conducta a seguir es ordenar la práctica de exámenes complementarios, facultad contemplada en los Artículo 13-7 y 36 del Decreto 2463 de 2001 , en vez de simplemente omitir la dolencia en el dictamen.

\section{CONCLUSIONES}

Como conclusión de este avance de investigaciones puede señalarse lo siguiente:

a. Que el derecho fundamental al debido proceso se aplica a todos los trámites en los que se vea comprometida la voluntad de la administración pública y en donde estén en juega los derechos constitucionales básicos de los ciudadanos

b. Que en relación con el trámite de calificación de la pérdida de la capacidad laboral este derecho debe ser tenido en cuenta y su desconocimiento es atentatorio de uno de los más elementales derechos de los afiliados.

\section{REFERENCIAS BIBLIOGRÁFICAS}

CORTE CONSTITUCIONAL DE COLOMBIA, sentencia c-010 (Corte Constitucional 2000).

CORTE CONSTITUCIONAL DE COLOMBIA, sentencia C-653 (Corte Constitucional 2001).

CORTE CONSTITUCIONAL DE COLOMBIA, sentencia t- 001 (Corte Constitucional 1993).

CORTE CONSTITUCIONAL DE COLOMBIA, sentencia c-406 (Corte Constitucional 1996).

CORTE CONSTITUCIONAL DE COLOMBIA, sentencia t- 345 (Corte Constitucional 1996).

CORTE CONSTITUCIONAL DE COLOMBIA, sentencia c-251 (Corte Constitucional 1997).

CORTE CONSTITUCIONAL DE COLOMBIA, sentencia t- 568 (Corte Constitucional 1999).

CORTE CONSTITUCIONAL DE COLOMBIA, sentencia t- 1319 (Corte Constitucional 2001).

CORTE CONSTITUCIONAL DE COLOMBIA, sentencia c- 671 (Corte Constitucional 2002) 
CORTE CONSTITUCIONAL DE COLOMBIA, sentencia C-506 (Corte Constitucional 2002). CORTE CONSTITUCIONAL DE COLOMBIA, sentencia t- 558 (Corte Constitucional 2003). CORTE CONSTITUCIONAL DE COLOMBIA, sentencia t- 786 (Corte Constitucional 2003). CORTE CONSTITUCIONAL DE COLOMBIA, sentencia T- 1142 (Corte Constitucional 2003). CORTE CONSTITUCIONAL DE COLOMBIA, sentencia T-597 (Corte Constitucional 2004). CORTE CONSTITUCIONAL DE COLOMBIA, sentencia c-1189 (Corte Constitucional 2005). CORTE CONSTITUCIONAL DE COLOMBIA, sentencia c- 731 (Corte Constitucional 2005). CORTE CONSTITUCIONAL DE COLOMBIA, sentencia T-031 (Corte Constitucional 2005). CORTE CONSTITUCIONAL DE COLOMBIA, sentencia T-222 (Corte Constitucional 2005). CORTE CONSTITUCIONAL DE COLOMBIA, sentencia T-746 (Corte Constitucional 2005). CORTE CONSTITUCIONAL DE COLOMBIA, sentencia C-929 (Corte Constitucional 2005). CORTE CONSTITUCIONAL DE COLOMBIA, sentencia C- 1189 (Corte Constitucional 2005). CORTE CONSTITUCIONAL DE COLOMBIA, sentencia T- 436 (Corte Constitucional 2005). CORTE CONSTITUCIONAL DE COLOMBIA, sentencia T-108 (Corte Constitucional 2007). CORTE CONSTITUCIONAL DE COLOMBIA, sentencia T-567 (Corte Constitucional 2008). CORTE CONSTITUCIONAL DE COLOMBIA, sentencia T-328 (Corte Constitucional 2008). CORTE CONSTITUCIONAL DE COLOMBIA, sentencia T-773 (Corte Constitucional 2009). CORTE CONSTITUCIONAL DE COLOMBIA, sentencia c-980 (Corte Constitucional 2010 CORTE CONSTITUCIONAL DE COLOMBIA, sentencia T-518 (Corte Constitucional 2011) CORTE CONSTITUCIONAL DE COLOMBIA, sentencia T-798 (Corte Constitucional 2011). CORTE CONSTITUCIONAL DE COLOMBIA, sentencia T-558 (Corte Constitucional 2011) CORTE CONSTITUCIONAL DE COLOMBIA, sentencia T-150 (Corte Constitucional 2013) CORTE CONTITUCIONAL DE COLOMBIA, sentencia SU- 250 (Corte Constitucional 1998).DECRETO, 2463 (2001 Art. 33-34). 